\title{
Los estudiantes universitarios guatemaltecos y su proyección social: la Universidad Popular (1923-1932)*
}

Recibido: 16 de enero de 2019

Aprobado: 7 de noviembre de 2019

Modificado: 18 de noviembre de 2019

Artículo de investigación científica

\section{Leticia GonZÁLEz SANDOval}

Investigadora del Instituto de Investigación y Proyección sobre el Estado de la Universidad Rafael Landívar (Guatemala).Correo electrónico: mlgonzalezs@url.edu.gt. La autora es doctora en Historia por la Universidad Pablo de Olavide (España). Entre sus publicaciones tenemos: "Entre la moralidad y el interés fiscal: el estanco de aguardiente en el periodo conservador", en Repensando Guatemala en la época de Rafael Carrera: el país, el hombre, y las coordenadas de su tiempo, Coord. Brian Connaughton, México: Universidad Autónoma Metropolitana-Ixtapalapa y Gedisa, 2015. Entre sus temas de interés se encuentran Historia de la educación, Historia agraria, Fiscalidad y estatalidad, y Construcción del Estado.

DOI: https://doi.org/10.15648/hc.38.2021.2814

Este artículo forma parte del proyecto "Los estudiantes universitarios guatemaltecos y su proyección social: la Universidad Popular (1923-1932)" financiación propia.

Esta publicación está bajo una licencia Creative Commons Reconocimiento-NoComercial 4.0 (c) BY-NC 
Los estudiantes universitarios guatemaltecos y su proyección social: la Universidad Popular (1923-1932)

\section{Resumen}

El artículo tiene como propósito comprender la creación y funcionamiento de la Universidad Popular de Guatemala en su primera década de existencia. La caída del gobierno de Manuel Estrada Cabrera contribuyó a la consolidación de un grupo de estudiantes universitarios, que inspirados en los planteamientos de la Reforma Universitaria de Córdoba (Argentina) y el pensamiento de José Vasconcelos, propusieron una política de "educación de masas" que vinculaba a la universidad con el pueblo, en especial con sus núcleos obreros urbanos. La experiencia no fue totalmente exitosa porque el idealismo romántico de los estudiantes colisionó con las condiciones sociopolíticas imperantes.

Palabras clave: educación popular, educación de adultos, Generación de 1920 (Guatemala), Universidades Populares, movimiento estudiantil, Guatemala.

\section{Guatemalan University Students and their Social Projection: The People's} University (1923-1932)

\section{Abstract}

The purpose of the article is to understand the creation and functioning of the People's University in its first decade of existence. The fall of the government of Manuel Estrada Cabrera contributed to the consolidation of a group of university students, who inspired by the University Reform of Córdoba (Argentina) and the thinking of José Vasconcelos, proposed a policy of "mass education" that linked the university with the people, especially with its urban working class. The experience was not entirely successful because the idealism of the students collided with the prevailing sociopolitical conditions.

Key words: popular education, Generation of 1920 (Guatemala), adult education, People's Universities, student movement, Guatemala

\section{Universitários guatemaltecos e sua projeção social: a Universidade Popular (1923-1932)}

\section{Resumo}

O objetivo do artigo é compreender a criação e o funcionamento da Universidade 
Popular em sua primeira década de existência. A queda do governo de Manuel Estrada Cabrera contribuiu para a consolidação de um grupo de estudantes universitários, que inspiraram as abordagens da Reforma universitária de Córdoba (Argenitna) e a interpretação feita José Vasconcelos, propôs uma política de "educação de massas" que ligava a universidade com o povo, especialmente com os núcleos de seus trabalhadores urbano. A experiência não foi totalmente bem-sucedida porque o idealismo dos estudantes colidiram com as condições sociopolíticas prevalecentes.

Palavras-chave: educação popular, Geração de 1920 (Guatemala), educação para adultos, Universidades Populares, movimento estudantil, Guatemala

\section{Étudiants universitaires guatémaltèques et leur projection sociale: 1'Université Populaire (1923-1932)}

\section{Resumé}

Cet article propose comprendre la création et le fonctionnement de l'Universidad Popular pendant la première décennie depuis sa création. La chute du gouvernement de Manuel Estrada Cabrera contribue à la consolidation d'un groupe d'étudiants universitaires qui proposera une politique dite «d'éducation des masses», inspirée par les principes de la réforme de l'université de Córdoba (Argentine) suivant l'interprétation faite par José Vasconcelos, visant à relier l'université avec le peuple (son noyau ouvrier urbain). L'expérience ne fut pas totalement réussi puisque l'idéalisme des étudiants se heurtent aux conditions sociopolitiques dominantes.

Mots clés: éducation populaire, Génération de 1920 (Guatemala), éducation des adultes, Universités Populaires, mouvement étudiant, Guatemala

\section{INTRODUCCIÓN}

En marzo de 1923 un editorial de El Imparcial, relacionado con la libre enseñanza, mencionó a la recién fundada Universidad Popular (UP) como un ejemplo de las posibilidades educativas fuera del sistema oficial. En un país con carencias en todos los ámbitos, esta iniciativa de un grupo de estudiantes universitarios fue bienvenida. La historia de la

1 El Imparcial fue un diario vespertino fundado en 1922 que pronto se volvió un referente de la vida política nacional. Clausurado por orden del gobierno de Orellana en 1926, volvió a circular tras la muerte de este. Su cierre definitivo tuvo lugar en 1985. 
UP de Guatemala tiene dos etapas, desde 1923 hasta 1932, y desde 1945 a la actualidad. Este artículo quiere ser una aproximación a la primera de ellas. Abarca, en consecuencia, una década que puede concebirse como el entreacto de dos dictaduras.

En Guatemala, la década de 1920 es un momento de apertura política. Una insurrección popular fue el principio del fin de la dictadura de Manuel Estrada Cabrera. En los años siguientes hubo un intento de democratización durante el cual el gobierno no reprimió la organización de obreros, artesanos y la clase media, quienes demandaban el reconocimiento de sus derechos políticos. Sin embargo, la oposición de la élite cafetalera y la jerarquía militar hizo tambalear al régimen y canceló las posibilidades de consolidación de un gobierno progresista. Al mismo tiempo, estos actores posibilitaron el surgimiento de una nueva dictadura, la de Jorge Ubico, más identificada con sus intereses.

La creación de la UP se enmarca en este contexto, aunada con la renovación del quehacer universitario concebido en la Reforma Universitaria de Córdoba (Argentina) y el pensamiento de José Vasconcelos en México. El contacto con una nueva visión del papel del estudiante universitario dio respuesta a la inquietud de un grupo de estudiantes, la llamada Generación de 1920, sobre el modo de enfrentar el "legado de ignorancia" que abatía a "las masas populares".

Así pues, el presente trabajo es un esfuerzo por sintetizar una etapa de la historia de Guatemala en la cual los ideales y prácticas políticas de un grupo de estudiantes universitarios, urbanos y ladinos, estimulados por Córdoba y Vasconcelos, intentaron llevar a cabo un proyecto imbuido con aires de modernización por medio del cual esperaban propiciar el tránsito de la población guatemalteca del rol de habitantes pasivos a ciudadanos conscientes de sus derechos. Este esfuerzo entró en ruta de colisión con las fuertes barreras estructurales existentes y la cultura política predominante.

2 "El destino de las escuelas libres", El Imparcial, Guatemala, 13 de marzo de 1923, 3. 


\section{LA CAÍDA DE UN DICTADOR}

En los primeros años del siglo XX Guatemala era gobernada por Manuel Estrada Cabrera. Este había llegado a la presidencia porque siendo Ministro del Interior fue nombrado Primer Designado. Con el asesinato de Reyna Barrios en febrero de 1898, Estrada Cabrera ganó "la lotería del poder"3: aunque ya no ocupaba un puesto en el gabinete, la Asamblea Legislativa no había revocado el decreto de sucesión y él lo hizo valer. Durante los siguientes 22 años ocupó la presidencia del país. En ese período enfrentó y desarticuló insurrecciones militares e invasiones armadas lideradas por civiles, y neutralizó a la oposición por diferentes medios ${ }^{4}$. El servilismo y la adulación se volvieron estrategias de supervivencia, en un ambiente opresivo impregnado por el miedo.

Aunque Estrada Cabrera se veía a sí mismo como promotor de la educación pública y protector de la juventud, su gobierno no se distinguió particularmente en ese aspecto. Si la educación primaria estaba abandonada, poco podía esperarse de la educación superior. En 1907 las cuatro escuelas facultativas de la universidad ${ }^{5}$ tenían un total de 232 alumnos, la mayoría de los cuales se encontraba en dos: Derecho y Notariado, y Medicina y Farmacia ${ }^{6}$. El número de estudiantes fue creciendo paulatinamente, pero en 1922 aún no llegaba al millar ${ }^{7}$. Sin

3 Mary Catherine Rendón, "Manuel Estrada Cabrera, Guatemalan President, 1898-1920" (Tesis de Doctorado, Oxford University, 1988), 117.

4 Aquí, como en otras partes del texto, se recurre al conocimiento común sobre el período. Dos tesis doctorales han sido fuentes básicas de información sobre el entorno político de la década: Mary Catherine Rendón, "Manuel Estrada Cabrera, Guatemalan President, 1898-1920", y Joseph A. Pitti, "Jorge Ubico and the Guatemalan Politics in the 1920" (Tesis de Doctorado, University of New Mexico, 1975).

5 En los primeros años del siglo XX, la educación superior siguió el modelo concebido en la Ley Orgánica y Reglamentaria de Educación de 1879, esto es, "escuelas facultativas" independientes. En abril de 1918, la Asamblea Legislativa creó por decreto la "Universidad Estrada Cabrera", cuyo objetivo fue "uniformar bajo un plan integral los estudios superiores y profesionales". En abril de 1924 se suprimió el nombre del expresidente y se le conoció como Universidad Nacional. En el decreto de autonomía universitaria de 1944 recuperó el nombre "Universidad Nacional de San Carlos" y en la Ley orgánica de 1945, se le denominó "Universidad de San Carlos de Guatemala". Augusto Cazali Ávila, Historia de la Universidad de San Carlos de Guatemala: época republicana, 1821-1944, $2^{\mathrm{a}}$ ed. (Guatemala: Editorial Universitaria, 2001), 196-214.

6 Guatemala, Secretaría de Instrucción Pública, Memoria de la Secretaría de Instrucción Pública presentada a la Asamblea Nacional Legislativa en 1907 (Guatemala: Tipografía Nacional, 1907), 8-9.

7 En 1923 había 819 estudiantes inscritos en las diferentes facultades de la universidad; en 1925 el número había aumentado a 1.621. Eugenia A. Phillips, "The Development of Modern Education in Guatemala” (Tesis de Maestría, University of Southern California, 1927), 66. 
embargo, en ellos se encontraba el germen de la insurrección que, combinada con otros factores externos e internos, derrocó al dictador.

El final de la Primera Guerra Mundial fue decisivo. Estrada Cabrera había retrasado el embargo de los bienes alemanes en Guatemala, postura insostenible en 1918, cuando finalmente cedió y confiscó propiedades y negocios. No obstante, su tardanza en tomar esta decisión parece haber influido en la política exterior de los Estados Unidos, que había empezado a impacientarse con el tirano.

El descontento también iba creciendo en el país. Manuel Cobos Batres y el arzobispo José Piñol y Batres iniciaron un movimiento conspirativo que encontró apoyo inusitado ${ }^{8}$. Los sermones de Piñol influyeron en la gente; además, provocaron su segundo exilio9. La respuesta tardía del gobierno a las necesidades de la población y los efectos económicos de los terremotos de 1917-1918 sumaron al descontento general. En la ciudad capital empezó a crecer el germen del Partido Unionista ${ }^{10}$.

Los descontentos que se aglutinaban alrededor de Cobos Batres y del arzobispo Piñol descubrieron que no estaban solos. La incipiente organización de trabajadores tomó forma en la Federación de Obreros de Guatemala, cuyo origen estuvo en los clubes obreros que Estrada Cabrera había alentado con fines electorales. Ambos grupos concluyeron que la unión era posible y necesaria ${ }^{11}$. Una vez superadas las desconfianzas mutuas, 20 obreros y artesanos firmaron el acta de organización del Partido Unionista, en diciembre de $1919^{12}$. A ellos se

8 Manuel Cobos Batres pertenecía a una de las familias de arraigo terrateniente de Guatemala. Era primo del obispo Piñol. Ambos enarbolaron principios liberales para combatir un gobierno liberal anquilosado que había negado esos principios en su práctica política.

9 Los discursos cívico-religiosos del arzobispo Piñol fueron fundamentales en el involucramiento de los obreros en la insurrección contra Estrada Cabrera. Renate Witzel de Ciudad (coord.), Más de 100 años del movimiento obrero urbano en Guatemala, tomo I (Guatemala: Asíes, 1991), 75-76.

10 El Partido Unionista recibió ese nombre porque, a 100 años de la Independencia, trató de revivir el ideario de la Unión Centroamericana.

11 Renate Witzel de Ciudad (coord.), Más de 100 años del movimiento obrero, 81-83.

12 Renate Witzel de Ciudad (coord.), Más de 100 años del movimiento obrero, 86. No debe extrañar el reducido número de obreros y artesanos que se involucraron en el movimiento unionista. En Guatemala la industria era embrionaria y se apoyaba fuertemente en procesos artesanales. En ese sentido, sería más apropiado hablar de talleres, no de fábricas. Una descripción de 1915 puede ayudar a comprender lo que se entendía por industria en esa época: "Hay, sin embargo, en la capital dos industrias principales: fabricación de calzado y ebanistería. La primera es de alta importancia. Indi- 
sumó otro actor: el estudiantado universitario. Manuel Cobos Batres no ignoraba su importancia en la organización partidaria. De esa cuenta, la dirigencia promovió la formación del Club Unionista de Estudiantes Universitarios. Fundado en enero de 1920, contó con la firma de 88 estudiantes de Derecho, Medicina y Farmacia ${ }^{13}$.

El derrocamiento de Estrada Cabrera fue el factor aglutinante de los tres actores mencionados. En la sublevación de marzo de 1920 los obreros estuvieron muy involucrados y aunque los estudiantes universitarios no participaron en los choques callejeros, su intervención fue decisiva en la insurrección ${ }^{14}$. Este grupo de estudiantes llegó a conocerse como la Generación de 1920. Algunos de ellos, Miguel Ángel Asturias por ejemplo, alcanzaron reconocimiento internacional décadas más tarde.

Una vez conseguido el objetivo, el Partido Unionista se resquebrajó. Los primeros en desencantarse fueron los obreros y sindicalistas, quienes fueron ignorados en la toma de decisiones políticas que siguieron al derrocamiento de Estrada Cabrera. El movimiento obrero se dispersó y un grupo asumió posturas radicales que llevaron a su aniquilamiento en la década siguiente. Los estudiantes siguieron otros derroteros. En cierta medida, ambos actores tomaron ventaja de la apertura de espacios que el gobierno de Carlos Herrera, quien sucedió a Estrada Cabrera en la presidencia, trajo consigo, incluidos los contactos con movimientos afines en el exterior.

viduos del país se dedican a ella, habiendo por término medio 15 o 20 talleres de primera clase y el calzado que en ellos se fabrica compite en todos los conceptos con el europeo y americano. Todo el trabajo en estos talleres se hace a mano. [...] una fábrica de sobres de papel. Este producto, de calidad bastante aceptable, llena todas las necesidades de la República [...]. En materia de ebanistería se hacen trabajos de verdadero mérito. Hay en la ciudad una fábrica de cerveza [...]. Industria que ha alcanzado incremento es la fabricación de imágenes [...] Existen fábricas de puros y cigarrillos [...]. Tenemos como industrias secundarias: fabricación de jabón y velas, fábricas de aguas gaseosas, trabajos en talabartería y en vidrio: una de tejidos de punto de media y otras de tinta de escribir [...]". J. Bascom Jones, ed., El libro azul de Guatemala, 1915 (New Orleans: Searcy \& Pfaff, 1915), 85.

13 Mary Catherine Rendón, "Manuel Estrada Cabrera", 251; Arturo Taracena Arriola, "El movimiento obrero y los estudiantes universitarios, 1920-1927”, Perspectiva No. 8 (enero-junio 1986): 65.

14 El papel de las mujeres en la insurrección correspondió a la época. En consecuencia, estuvo vinculado a su condición doméstica. No había expectativas sobre su participación política y no estaban totalmente incorporadas al movimiento obrero. A pesar de ello, participaron en la gran marcha del 11 de marzo de 1920 y durante la insurrección curaron heridos y distribuyeron alimentos. Lorena Carrillo Padilla, Luchas de las guatemaltecas del siglo XX. Mirada al trabajo y la participación política de las mujeres (Guatemala: Ediciones del Pensativo, 2004), 141-149. 


\section{La influencia de Córdoba y México en los estudiantes GUATEMALTECOS}

La participación en la insurrección de marzo y abril de 1920 dio a los estudiantes una nueva visión de lo que podían hacer. El momento era oportuno para lograr una organización propia y siguiendo esa inquietud, delegados de las asociaciones Juventud Médica, El Derecho y Escuela de Farmacia fundaron el 22 de mayo de ese año la Asociación de Estudiantes Universitarios (AEU). Eran, si no los mismos, casi todos los que habían estado involucrados en el Club Unionista de Estudiantes Universitarios. La constitución de la AEU significó el alejamiento de la política partidista, pero no del interés en la participación política ${ }^{15}$.

Los estudiantes guatemaltecos no habían sido influenciados directamente por el movimiento de reforma universitaria que había recorrido América del Sur en las dos primeras décadas del siglo XX. Lo que habían hecho era participar en el derrocamiento de una dictadura. En otras palabras, su despertar político y su actitud insurgente tuvieron lugar en el epicentro de un cambio de régimen. Lo anterior abrió posibilidades para intercambiar ideas sobre la universidad y su vinculación con la sociedad de la cual era producto.

En Guatemala, la Universidad Nacional era elitista en la medida en que su acceso era restringido a cierto sector de las clases medias y altas urbanas, pero no estaba ligada a la Iglesia católica; la reforma liberal del siglo XIX había roto ese vínculo. Además, hay que tener presente que en el país solo funcionaba un centro de educación superior, por lo que su proyección social también era limitada. A esto hay que añadir la escasa cobertura. En consecuencia, no existía ni se promovía una vinculación con la sociedad, característica que la acerca a las carencias que la Reforma de Córdoba denunció ${ }^{16}$. Esta será una de las preocupa-

15 Arturo Taracena Arriola, "El movimiento obrero", 66; Virgilio Álvarez Aragón, Conventos, aulas y trincheras: universidad y movimiento estudiantil en Guatemala, tomo I (Guatemala: FLACSO, 2002), 160-161.

16 El postulado de la reforma universitaria propuesta en Córdoba relacionado con las Universidades Populares es el que se refiere a la extensión universitaria y al fortalecimiento de la función social de la universidad. En otras interpretaciones correspondería a la vinculación de la universidad con el pueblo. Carlos Tünnermann Bernheim, "La reforma universitaria de Córdoba", Educación Superior y Sociedad Vol. 9, No.1 (1998): 118-119. Ricardo Melgar Bao, "Las universidades populares en América Latina, 1910-1925”, Estudios No. 11-12 (enero-diciembre 1999): 43-44. 
ciones de los estudiantes, que encontraron las respuestas que buscaban fuera del país.

Después de veinte años de aislamiento intelectual, la primera oportunidad vino de México, que se encontraba en plena efervescencia revolucionaria. José Vasconcelos, Rector de la Universidad Nacional Autónoma de México, promovió un Congreso Internacional de Estudiantes. Delegados de 77 universidades y de cinco federaciones estudiantiles de América del Sur llegaron al evento en septiembre de 1921. Miguel Ángel Asturias era uno de los tres representantes guatemaltecos. El discurso inaugural de Vasconcelos estuvo inspirado por el pensamiento de la Reforma Universitaria de Córdoba ${ }^{17}$. Entre los temas abordados en el congreso se encuentran dos fundamentales: la función social del estudiante y el mejor método para ejercerla ${ }^{18}$. En la adopción de la extensión universitaria como estrategia para la acción social puede apreciarse la influencia de Córdoba:

“[...] La Extensión Universitaria es una obligación de las asociaciones estudiantiles, puesto que la primera y fundamental acción que el estudiante debe desarrollar en la sociedad es difundir la cultura, que de ella ha recibido, entre quienes la han menester. [...] que es una obligación de los estudiantes el establecimiento de universidades populares, que estén libres de todo espíritu dogmático y partidista y que intervengan en los conflictos obreros inspirando su acción en los modernos postulados de justicia social. El Congreso [...] sanciona como una necesidad, para las Universidades donde no se hubieren implantado, la adopción de las siguientes reformas: 1. Participación de los estudiantes en el gobierno de las universidades. 2. Implantación de la docencia libre y la asistencia libre. [...] Para realizar estos puntos el Congreso considera obligatoria la acción inmediata de los estudiantes en sus respectivos países"19.

17 Arturo Taracena Arriola, "Miguel Ángel Asturias y la búsqueda del 'alma nacional' guatemalteca. Itinerario político", en Miguel Angel Asturias, París 1924-1933. Periodismo y creación literaria, coord. Amos Segala (Madrid: ALLCA XX, 1988), 681.

18 "Resoluciones del Primer Congreso Internacional de Estudiantes reunido en la ciudad de México del 20 de setiembre al 8 de octubre de 1921", Repertorio Americano Vol. 3 No. 27 (27 de febrero de 1922): 375 .

19 "Resoluciones del Primer Congreso Internacional: 375-376. 
Los universitarios guatemaltecos también fueron influenciados por el panamericanismo ${ }^{20}$. Baste recordar la importancia que estaba adquiriendo el capital estadounidense en Guatemala para entender por qué la idea de realizar en el país un congreso panamericano de estudiantes fue apoyada por los unionistas. Lo interesante es que los estudiantes acogieron la idea sin cuestionarla, lo cual puede ser un indicio de su maleabilidad ideológica, pero también de su avidez por estar al tanto de las corrientes predominantes de la época. En otras palabras, estaban abiertos a la experiencia mexicana pero no se habían decantado totalmente por ella ${ }^{21}$. Prueba de lo anterior es su apertura a la idea del panamericanismo promovido por los Estados Unidos.

\section{La convocatoria para realizar el I Congreso Panamericano de Estudiantes} se lanzó en junio y este se llevó a cabo en septiembre de 1921, como parte de las celebraciones del primer centenario de la Independencia de Centroamérica. Seis fueron los temas propuestos: a) Medios de hacer efectiva la Liga de Estudiantes Panamericanos, b) la Liga de Estudiantes Panamericanos ante la política internacional americana, c) el estudiante como factor en el desenvolvimiento social, d) representación de los estudiantes en los cuerpos directivos de las facultades, e) sistema universitario que debe adaptarse en América, y f) unificación de programas universitarios $^{22}$. Aunque en los dos primeros se puede notar la influencia temática del congreso, en los tres últimos es palpable la influencia de Córdoba.

Las conclusiones sobre el papel de los estudiantes como factor para el desenvolvimiento social son esclarecedoras pues se les atribuyen dos

20 Aquí se hace referencia al panamericanismo promovido por los Estados Unidos. Es sabido que la intervención de ese país en Latinoamérica tiene sus raíces en la Doctrina Monroe y en el corolario de Roosevelt a esa doctrina. Se inserta además en la carrera imperialista mundial, la expansión del comercio, el reacomodo geopolítico posterior a la I Guerra Mundial y el surgimiento de los Estados Unidos como poder regional y mundial. Mark A. Burkholder, Monica Rankin y Lyman L. Johnson, Exploitation, Inequality and Resistance: A History of Latin America since Columbus (New York y Oxford: Oxford University Press, 2018), 258-273.

21 Epaminondas Quintana, uno de los miembros de la Generación de 1920, al hacer referencia a la influencia de Vasconcelos en la concepción de la UP, dice: "Yo no entendía mucho de eso. Sabía sí que Vasconcelos en México estaba sacando a la Universidad a la calle y que la Extensión Universitaria prometía ciencia, arte y alegría a las masas populares". "Cómo nació el ubérrimo árbol", El Imparcial, 1 de marzo de 1963, 11.

22 Citados por Epaminondas Quintana, Historia de la generación de 1920 (Guatemala: Tipografía Nacional, 1971), 158. 
funciones: "1º Una función interna de propio mejoramiento; y $2^{\circ}$ Una función externa, como miembro de la sociedad en que vive, encaminada a orientar a esta última hacia la liberación intelectual, moral y física de las masas populares" 23 . Asimismo, la discusión sobre el sistema universitario incluyó como una de las reformas necesarias la adopción de la extensión universitaria ${ }^{24}$.

La experiencia de los participantes en ambos congresos dio pie a que los estudiantes que eran diputados presentaran un plan de organización de la Universidad Nacional en la Asamblea Legislativa en $1922^{25}$, que incluyó la representación estudiantil en el consejo superior. Asimismo, sugirió la creación de "un departamento de extensión universitaria, para vincular profundamente la universidad al pueblo ${ }^{26}$. Aunque la iniciativa no prosperó, es evidente que el espíritu de la Reforma Universitaria había dejado huella en los estudiantes.

\section{La fundación de la Universidad Popular, logros y LIMITACIONES}

"De todas las hazañas y logros de la generación de estudiantes de 1920 (enfrentarse a la secular dictadura cabreriana; participar axialmente en la campaña unionista; hacer el Primer Congreso Centro-Americano de Estudiantes; fundar la AEU; hacer la revista Studium, sacar la Huelga de Dolores en gran parada hacia las calles; hacer del No Nos Tientes un periódico formidable; [...] editar el semanario Claridad, fundar el Grupo Vida; hacer La Chalana; editar la revista Ensayos en París, etc.) nada nos satisface más y nos recordará mejor a las generaciones venideras que el haber plantado ese árbol hoy frondoso que se llama Universidad Popular" ${ }^{27}$.

23 Epaminondas Quintana, Historia de la generación de 1920, 165.

24 Epaminondas Quintana, Historia de la generación de 1920, 171.

25 Entre ellos se encontraba Carlos Enrique Larraondo, quien fue uno de los delegados de Guatemala en el Congreso Panamericano de Estudiantes Universitarios.

26 Moisés Castro Morales y Jorge del Valle Matheu, "El movimiento reformista en las universidades nacionales y populares de Centro América", en La reforma universitaria, tomo II, comp. Gabriel del Mazo (La Plata: Centro de Estudiantes de Ingeniería, 1941), 199.

27 Epaminondas Quintana, "Cómo nació el ubérrimo árbol", 11. Studium fue la revista de la AEU en esos años. El No nos tientes es un semanario que se publica como antecedente de la Huelga de Dolores, desfile bufo universitario que precede a la Semana Santa; se caracteriza por su espíritu festivo y crítico. "La Chalana" es una canción crítica e irreverente, escrita por algunos de los fundadores de la 
Con esas palabras Epaminondas Quintana inició el texto con el cual celebró los 40 años de fundación de la Universidad Popular. Es un resumen de lo que supuso para los jóvenes de esa época asumir y ejercer sus deberes y derechos ciudadanos después de 22 años de dictadura y, al mismo tiempo, un reflejo de los intereses diversos que los caracterizaron. Fue una generación multifacética que escribía, fundaba periódicos, revistas y semanarios, tenía inquietudes políticas y cierto sentido de responsabilidad social que cuajó en la UP.

Aunque los fundadores de la UP reconocieron la influencia de Ricardo Arenales (Porfirio Barba Jacob) en la idea germinal de la universidad, no se puede negar que los aprendizajes en los congresos internacionales de estudiantes celebrados en esos días tuvieron un papel preponderante. Según Quintana, en 1922 Miguel Ángel Asturias, Carlos Fletes Sáenz, David Vela y él mismo, discutieron los cinco principios que regirían el Instituto de Cultura Popular, origen de la UP. Estos principios "resumían los anhelos del estudiantado de entonces: 1) civismo, por los afanes que acabábamos de pasar (la caída de la más secular de las tiranías de C. A. [Centro América]), 2) derechos, 3) higiene, 4) artesanías y 5) cultura general" 28 .

\section{Imagen 1}

Fundadores de la Universidad Popular de Guatemala. De izquierda a derecha: Epaminondas Quintana, David Vela, Miguel Ángel Asturias, Carlos Fletes Sáenz

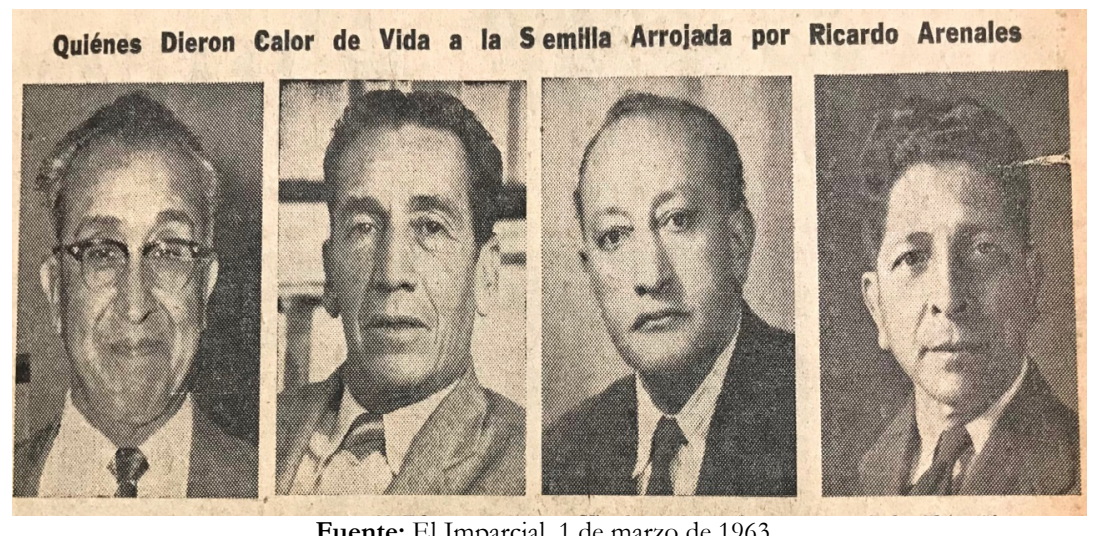

Fuente: El Imparcial, 1 de marzo de 1963.

Universidad Popular con motivo de la Huelga de Dolores.

28 Epaminondas Quintana, "Cómo nació el ubérrimo árbol”, 11. 
Sin embargo, el texto que se reconoce como el fundamento de la UP fue publicado por Clemente Marroquín Rojas, David Vela, Miguel Ángel Asturias y Alfonso Orantes en un editorial de la revista Cultura dedicado a la educación superior, publicado en septiembre de ese mismo año ${ }^{29}$. Ellos hicieron una crítica a la concepción de la universidad de la época y agregaron que debía salir de las "paredes claustrales" y "elevar el nivel cultural del pueblo"30. Así interpretaron la extensión universitaria promovida bajo el ideario de Córdoba y México. No podía ser de otra manera en un país en el cual la tasa de analfabetismo rondaba el 90 por ciento $^{31}$.

Para lograrlo, propusieron tres fines. El primero era la "desanalfabetización de las masas", cuya necesidad plantearon en los siguientes términos:

"Ya que una civilización no puede improvisarse, precisa crear desde un principio bases de aptitud, laborando en el terreno virgen de nuestra general ignorancia los surcos que reciban mañana una superior preparación. Enseñar a nuestro pueblo a balbucear en el silabario la promesa de un futuro mejor, dignificado por la certeza de un sentimiento consciente. Esta es una labor ardua, apostólica, en que se harán necesarias una paciencia y una firmeza que resistan duras pruebas y necesarios desencantos" ${ }^{\prime 3}$.

29 Clemente Marroquín Rojas tuvo una destacada carrera política; fue miembro de la Asamblea Constituyente en 1944, aunque después pasó a la oposición. Ocupó la Vicepresidencia de la República en el período 1966-1970. En la década de 1920 fundó el diario vespertino La Hora. Al igual que Marroquín Rojas, David Vela se graduó de abogado y fue Constituyente en 1944. En la década de 1920 fue jefe de redacción de El Imparcial y, tras el asesinato de Alejandro Córdova, asumió la dirección del diario. Es autor de varios libros y ensayos sobre literatura e historia de Guatemala. Alfonso Orantes también se graduó de abogado, pero su inclinación eran las letras. Fue catedrático universitario y colaboró en las revistas Alero y Universidad de San Carlos. Carlos Fletes Sáenz, nicaragüense radicado en Guatemala, estudió medicina, profesión que ejerció en el oriente del país. Fue uno de los más entusiastas profesores de la Universidad Popular. La carrera literaria de Miguel Ángel Asturias es sobradamente conocida. Epaminondas Quintana, Historia de la generación de 1920, 402, 426, 437.

30 "Universidad Popular: tres consideraciones sobre la universidad clásica cerrada", Cultura Vol. 1 No. 3 (septiembre 1922): 2.

31 Guatemala, Ministerio de Fomento, Censo de la población de la República, levantado el 28 de agosto de 1921, parte I (Guatemala: Talleres Gutenberg, 1924), 71.

32 "Universidad Popular: tres consideraciones", 3. 
Esta fue la lectura que el grupo hizo del "problema de educación nacional” que la Secretaría de Instrucción Pública planteó como “[...] la gran incógnita a despejar [...] pues en el estado de obscuridad mental en que hoy se encuentran, cuando no son elementos retardatorios $u$ obstruccionistas, desempeñan una función secundaria y primitiva en el movimiento del progreso material y espiritual"'33. Ambas aproximaciones al analfabetismo comparten el mismo prejuicio, pero mientras los estudiantes lo hacen desde una visión romántica, la Secretaría no tiene reparos en reproducir una concepción discriminatoria que había sido parte del discurso oficial desde el triunfo de la reforma liberal de 1871.

El segundo fin de la propuesta estaba centrado en la divulgación científica. Aquí consideraron que lo importante era el "cultivo de la inteligencia”, dadas las limitaciones que los horarios de trabajo imponían a la instrucción de obreros y artesanos. El tercero, denominado "formación del alma nacional", era una de las preocupaciones fundamentales del grupo; su significado estaba ligado a una concepción de patria que incluía superar la ignorancia y que tenía como fin último el bienestar general $^{34}$. Ideas similares se expusieron en un editorial de Studium, que planteó la renovación del país basándose en una premisa a primera vista simple y alcanzable: "impartiendo la enseñanza en el pueblo [...]"35. Para los universitarios, el acceso a la educación era la solución de todos los problemas sociales $^{36}$.

Del proyecto estuvo ausente, en términos de la época, "el problema del indio". En ese sentido, debe recordarse que la instrucción que los estudiantes querían impartir estaba dirigida en primer lugar a obreros y artesanos que habitaban los centros urbanos ${ }^{37}$. No plantearon la

33 Guatemala, Memoria de la Secretaría de Educación Pública, presentada a la Asamblea Nacional Legislativa en sus sesiones ordinarias de 1924 (Guatemala: Tipografía Nacional, 1924), 44.

34 "Universidad Popular: tres consideraciones", 3-4.

35 "Página editorial", Studium Vol. 2 Nos. 2 y 3 (febrero-marzo 1923): 1.

36 Cuarenta años más tarde, Epaminondas Quintana dirá: "En aquel entonces, creíamos que la letra redime por sí sola [...] soñamos que diciéndole al pueblo cuáles eran sus deberes ciudadanos, él iba a transformarse de manada de ovejas en grupos de ciudadanos perfectos". "Cómo nació el ubérrimo árbol", 11.

37 Durante su estancia en París, Miguel Ángel Asturias publicó en El Imparcial varios artículos en los que se refirió a la población objetivo de la UP. Por ejemplo: "En ella se preparan las generaciones obreras 
alfabetización de los indígenas ni estrategias de asimilación; más bien esperaban que la población analfabeta, sin importar su etnia, se acercara a la UP. Creían que "despertando la necesidad de aprender" y haciendo un llamado a los finqueros surgirían los centros de alfabetización en el área rural ${ }^{38}$.

La UP contó con el respaldo de por lo menos un diputado de la Asamblea Nacional Legislativa, quien presentó una moción para crear una renta a su favor. La propuesta era gravar cada quintal de café exportado. Otra iniciativa planteó gravar los artículos suntuarios ${ }^{39}$. Ninguna de las dos fue aprobada; la UP pudo funcionar gracias al apoyo del Gobierno de la República, de la Municipalidad de Guatemala y de alrededor de 200 donantes particulares que contribuían con cantidades que oscilaban entre 25 y 500 pesos mensuales ${ }^{40}$.

Los estudiantes y jóvenes profesionales sostenían reuniones para ponerse de acuerdo en una amplia variedad de temas, que iban desde la recolección de fondos y la búsqueda de locales en donde empezaría a funcionar la UP, hasta la elaboración de los planes de enseñanza ${ }^{41}$. Asimismo, no dudaron en escribirle a José Vasconcelos para solicitarle consejos sobre cómo debía manejarse la UP en el país; le pidieron además libros para la futura biblioteca ${ }^{42}$.

Aunque los involucrados en la creación de la UP reconocieron como fecha de su fundación el 20 de agosto de $1922^{43}$, fue el 1 de marzo de

del mañana [...]”; “[...] mi mayor ambición al volver es ocupar mi puesto entre mis alumnos obreros [...]"; "Se llama a la gente de trabajo a esta universidad y se le enseña lo que ha de servirle [...]". Miguel Ángel Asturias, París 1924-1933, 18, 75, 252.

38 "La Universidad Popular. Nobilísima campaña de la entidad que hoy se funda", El Imparcial, 1 de marzo de 1923, 6. Según el diario, el contenido de la nota se basaba en una conversación sostenida con uno de los organizadores de la UP.

39 El Imparcial, 6 de marzo de 1923, 1 y $8 ; 8$ de marzo de 1923, 3.

40 Universidad Popular, Memoria de los trabajos efectuados en la Universidad Popular de Guatemala, hasta el 31 de diciembre de 1924 (Guatemala: Talleres Selca, 1924), 8-10.

41 El apoyo de las mujeres consistió en la creación del Consejo de Señoras de la UP. "Extracto de las primeras actas de la Universidad Popular", El Imparcial, 1 de marzo de 1963, 11 y 17.

42 Según Arturo Taracena, el embajador mexicano, actuando como enlace de Vasconcelos, quien en ese momento ya era Secretario de Instrucción Pública de México, estuvo muy involucrado en la fundación de la UP. "El movimiento obrero", 69.

43 Ese día, un grupo se reunió para integrar la junta provisional de organización que tenía como objetivo llevar a cabo los trabajos preparatorios para la fundación de la Universidad Popular. Entre los 
1923 cuando se inauguró el año escolar, en la sede de la Asociación de Estudiantes Universitarios. La puesta en marcha de la UP puede interpretarse como una crítica velada al sistema educativo oficial. Quizás por eso el Secretario de Instrucción Pública no asistió al acto. Estuvieron presentes el Rector de la Universidad Nacional, el Ministro de Gobernación y comisiones de los centros obreros ${ }^{44}$. El acuerdo gubernativo que aprobó los estatutos y reconoció la personalidad jurídica de la UP fue emitido el 30 de noviembre de ese año.

\section{Imagen 2}

Sede de la Asociación de Estudiantes Universitarios, 1923 ("Edificio de la Asociación de Estudiantes Universitarios, donde tiene su asiento la Universidad Popular que se inaugura esta noche"

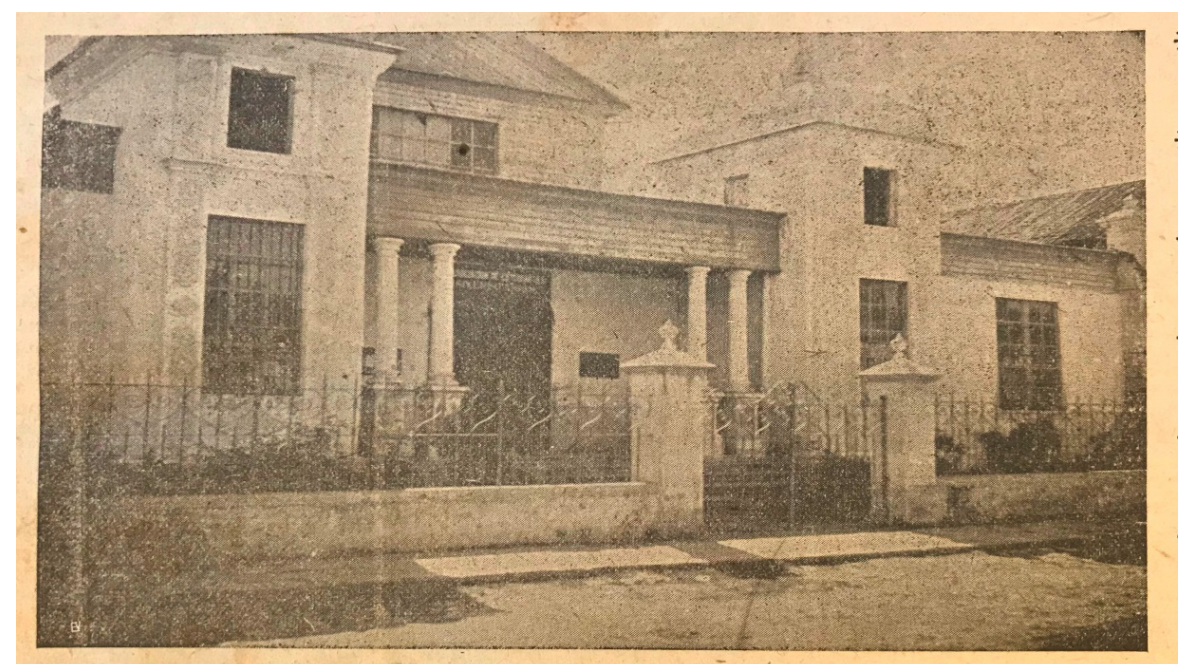

Fuente: El Imparcial, 1 de marzo de 1923.

El sistema educativo formal contaba con escuelas nocturnas para adultos; si la UP quería marcar la diferencia, tendría que hacerlo yendo más allá de la mera alfabetización. De ahí que la universidad se planteara

asistentes estuvieron Miguel Ángel Asturias, Carlos Flete Sáenz, Alfonso Orantes, Ricardo Arenales, Rafael Lassepas, David Vela y Rufino Guerra Cortave. "Extracto de las primeras actas de la Universidad Popular", 11.

44 El Imparcial, 1 de marzo de 1923, 1; 2 de marzo de 1923, 1. El diario apoyó a la UP desde la fundación de esta. Por ejemplo, en la primera página de su edición del 1 de marzo se lee: "Inauguran hoy los estudiantes universitarios la institución más bella y útil para Guatemala: la Universidad Popular de que todos debemos esperar la eficaz campaña de desanalfabetización y a la que todos debemos ayudar". Al igual que los promotores de la UP, El Imparcial creía que una población alfabeta contribuiría al adelanto del país. "La Universidad Popular. Nobilísima campaña de la entidad que hoy se funda", 6. 
tres objetivos: el primero se refería a la enseñanza de nociones elementales de lectura y escritura y de las reglas básicas de la aritmética. Así cubría el contenido de un cursillo de alfabetización. El segundo objetivo pretendía ensanchar los conocimientos generales de los hombres y mujeres que llegaran a sus aulas, y el tercero se proponía la difusión de nociones de instrucción cívica, moral e higiene ${ }^{45}$. La enseñanza del civismo era una de las preocupaciones de los estudiantes universitarios; ellos creían que la cultura cívica salvaría a las masas de seguir ciegamente a líderes de ocasión ${ }^{46}$.

La instrucción cívica, la moral y la higiene no eran una novedad curricular. Las tres eran parte de los programas detallados de la escuela primaria complementaria, aglutinadas bajo la denominada educación moral $^{47}$, pero debido al acceso limitado a esos tres grados, es posible suponer que las nociones sobre deberes y derechos ciudadanos, en su versión escolar, eran desconocidas para la mayoría de la población adulta.

Entender por qué era importante la instrucción cívica es fácil si se toma en cuenta que la generación de 1920 había crecido bajo una dictadura. Además, hay que tener presente que el primer interludio democrático de la década no rebasó los dos años. Carlos Herrera fue depuesto por un golpe de Estado en diciembre de 1921. Le sucedió en el poder el general José María Orellana, a quien se enfrentaron estudiantes universitarios y trabajadores debido a las concesiones que hizo a compañías estadounidenses. Hubo un levantamiento general que fracasó, se implementó una ley marcial y se suprimieron cinco periódicos. Además, Orellana decretó el cierre de la Universidad Nacional en abril de 1924, pero la

45 Universidad Popular, Prospecto de la Universidad Popular (Guatemala: Tipografía Nacional, 1923), 3.

46 Gustavo Martínez Nolasco, "El estudiante como factor social", Studium Vol. 1 No. 7 (noviembre-diciembre 1921): 28, citado por Joseph A. Pitti, "Jorge Ubico and the Guatemalan Politics", 208. Años más tarde, Miguel Ángel Asturias dijo: "La meta de la Universidad Popular fue enseñarles a leer y escribir [a los obreros], pero aunque esto era muy importante, lo que nos interesaba más era enseñarles al mismo tiempo cuáles eran sus derechos y obligaciones según la Constitución [...] la gente era demasiado ignorante para defender derechos y libertades que no sabían siquiera que existían". Rita Guibert, "Miguel Angel Asturias", en Seven Voices: Seven Latin American Writers Talk to Rita Guibert (New York: Alfred A. Knopf, 1973), 129.

47 Guatemala. Secretaría de Instrucción Pública, Programas detallados para las escuelas de enseñanza complementaria de la República de Guatemala (Guatemala: Tipografía Nacional, 1922), 7, 25, 59. 
educación superior continuó en la modalidad de escuelas facultativas. En ese ambiente políticamente convulso, al que hay que agregar una crisis económica producto de una nueva caída de los precios del café en el mercado mundial, se estaba tratando de impulsar a la UP.

El programa de estudios de la UP se dividió en dos secciones, elemental y superior. La primera se subdividió también en dos: para analfabetos y para "medio-analfabetos". En ambas la enseñanza se concentraba en lectura y escritura, gramática, aritmética, e instrucción cívica, moral e higiene. La sección superior estaba dividida en seis ciclos y en ella solo se admitía a quienes ya supieran leer y escribir. Aquí es donde se puede apreciar el alcance del proceso educativo que los fundadores de la UP habían concebido. Se trataba de ciclos simultáneos y los estudiantes podían empezar por el que tuviera un contenido que les atrajera más. No había exámenes ni calificaciones, aunque se esperaba que los inscritos abarcaran todas las materias incluidas en los seis ciclos en un lapso de año y medio.

El primer ciclo era básico, la diferencia con la sección elemental era la inclusión de lecturas escogidas. El tercero tenía una orientación comercial; los estudiantes podían aprender teneduría de libros, taquigrafía, caligrafía e inglés y francés. El quinto ciclo estaba dedicado a la agricultura y el sexto, llamado "de adorno", a las humanidades; ahí tenían cabida lecciones de historia, geografía y filosofía. Como era de esperar, los seis ciclos tenían en común la enseñanza de moral, higiene e instrucción cívica ${ }^{48}$. El papel de la Secretaría de Instrucción Pública se reducía a practicar una prueba de suficiencia que los alumnos del ciclo elemental hacían ante el inspector nombrado por el Departamento Pro-Alfabeto ${ }^{49}$.

Los trabajadores recibían clases por la noche, de 19:00 a 22:00 horas. En el edificio de la UP solo se atendía la sección elemental (alfabetización). La UP contaba además con aulas en la Facultad de Derecho y otras proporcionadas por establecimientos educativos privados en el centro de la ciudad capital. El entusiasmo inicial sirvió de estímulo para

49 Universidad Popular, Reglamento y programas para las escuelas de analfabetos de la Universidad Popular de Guatemala (Guatemala: s.e., 1923), 3. 
que varios particulares alfabetizaran en sus casas de habitación; ellos mismos eran los profesores: "En la casa de don Eliseo Paiz asistieron término medio 20 personas; en la casa de don Max Aldana, cinco. En casa del licenciado Arturo Rosal, como resultado de la dedicación del mismo, aprendieron cinco obreros" $" 50$.

Tanto estos profesores, llamados adherentes, como los de curso, recibieron un folleto con instrucciones mínimas que describían su función. Estas tomaban en cuenta que la mayoría de ellos no tenía experiencia en la enseñanza. En el caso de los primeros, se insistía en lo siguiente: "Enseñe usted a leer y escribir, no nos importa, por ahora, el libro y método que emplee, enseñe como a $\mathrm{Ud}$. le enseñaron, lo que deseamos y precisa es que enseñe"

En ese pequeño folleto quedó enunciada la intención de la UP en esa primera etapa: "Enseñe a personas de cualquier edad, condición o estatuto, sin esperar pago ni recompensa. A la obra nacional de desanalfabetizar contribuya con veinte minutos diarios en la sala de su casa [...] no nos importa el lugar, lo que deseamos y precisa es que enseñe" ${ }^{52}$. A los profesores de curso se les pidió que la enseñanza fuera sencilla y esencialmente práctica y que no complicaran a los alumnos "con metafísica, que no hace más que confundirlos" 53 .

En su primer año, la UP contó entre sus docentes a los fundadores, quienes contagiaron su entusiasmo a sus compañeros de estudios. Miguel Ángel Asturias y Alfonso Orantes tenían a su cargo las clases de lectura y escritura; David Vela, las de aritmética práctica y Epaminondas Quintana, las de higiene. La instrucción cívica estaba a cargo de Rafael Castellanos y Manuel de León Cardona; José Luis Balcárcel enseñaba taquigrafía, Rafael Pérez de León, dibujo lineal. Rufino Guerra Cortave, Alfonso Carrillo, y Marco Antonio Asturias eran parte del claustro.

50 Universidad Popular, Memoria de los trabajos efectuados, 13. Como puede inferirse, los métodos de enseñanza no fueron homogéneos. Los docentes en la UP eran estudiantes universitarios y los entusiastas que abrieron sus casas para impartir cursos de alfabetización no eran necesariamente maestros. Lamentablemente, la UP no resguardó sus archivos, posiblemente desaparecieron en la década en que estuvo cerrada, lo cual hace muy difícil abordar la cultura escolar, por ejemplo, o precisar datos sobre sus alumnos.

51 Universidad Popular, Guía de profesores adherentes (Guatemala: Tipografía Nacional, 1923), 1.

52 Universidad Popular, Guía de profesores adherentes, 1.

53 Universidad Popular, Guía de profesores de curso (Guatemala: Tipografía Nacional, 1923), 1. 
Profesionales como Francisco Orla, Carlos Federico Mora, Carlos Bauer Avilés y Alberto Velásquez también enseñaron ahín ${ }^{54}$ Algunos de ellos, como Asturias, quien se instaló en París, dejaron pronto la enseñanza. Otros, una vez graduados, se dedicaron a la práctica privada o fueron absorbidos por la burocracia estatal ${ }^{55}$.

Quizás a eso se deba que con el paso del tiempo el esfuerzo que había supuesto pensar la UP como una opción educativa para los trabajadores y la puesta en marcha de la institución fuera decayendo. Además, la respuesta de la población no fue la esperada; de tal suerte, en 1924 solo 92 obreros aprendieron a leer y a escribir. En el interior de la República solo funcionaron dos sucursales, una en Amatitlán, en la que se alfabetizaron 18 personas, y otra en Huehuetenango, en la que se inscribieron 61 personas ${ }^{56}$. Carlos Federico Mora atribuyó el poco desarrollo alcanzado en el interior al "factor de la masa analfabeta que, mucho más numerosa, reacia como es por naturaleza a la instrucción y diseminada en fincas y aldeas, no concurrió como debiera haberlo hecho, al llamado que se hizo" 57 .

Esta aseveración muestra, por un lado, uno de los prejuicios comunes en la época: a la población del área rural, mayoritariamente indígena, no le interesaba la educación formal; y por el otro, el desconocimiento que tenían los habitantes de la ciudad (en este caso, los estudiantes y profesionales) de las condiciones de vida en el campo. En la década de 1920 la habilitación para trabajar en las fincas de café impedía per se la asistencia a la escuela. Tan permeado estaba el sistema educativo por la economía cafetalera que el Estado delegó a los finqueros la educación de los trabajadores; para cortar café no era necesario saber leer y escribir;

54 "Los primeros profesores de la Universidad Popular", El Imparcial, 1 de marzo de 1963, 11; Miguel Ángel Asturias, París 1924-1933, 540, nota de Gerald Martin.

55 Epaminondas Quintana reconoció como un error imperdonable la alineación de los estudiantes con los grupos de poder detrás de cada elección presidencial. Los estudiantes o profesionales reclutados de ese modo ocupaban después "[...] subsecretarías, direcciones generales, magistraturas, diputaciones y otros puestos bien remunerados". Citado por Virgilio Álvarez Aragón, Conventos, aulas y trincheras, 191-192.

56 Universidad Popular, Memoria de los trabajos efectuados, 13-18. La UP no alcanzará la cobertura que sus fundadores esperaron. Asturias se refirió a las "reducidas cifras" de la memoria correspondiente a 1925, las que "dejan en el espíritu la impresión de que no tenemos remedio". Miguel Ángel Asturias, "Memoria de la Universidad Popular", en París 1924-1933, 136-137.

57 Universidad Popular, Memoria de los trabajos efectuados, 44. 
en consecuencia, la alfabetización no era prioritaria y lo que se requería era la castellanización.

En lo que respecta a la divulgación científica, esto es, la sección superior de enseñanza en la UP, su radio de acción fue aún más limitado ya que el número de alumnos fue menor. Al igual que en el departamento pro-alfabeto (es decir, la sección elemental de la UP) la escasez de profesores afectó el desempeño. Lo mismo sucedió con las conferencias: resultaba difícil conseguir disertantes ${ }^{58}$.

Puede ser que este fracaso aparente fuera la razón detrás de los artículos que Miguel Ángel Asturias, desde París, enviaba con frecuencia a El Imparcial. En ellos, defendía a la UP y exhortaba a los trabajadores a no abandonar la institución que había sido creada pensando en ellos: "El obrero que no ayuda a la Universidad Popular, destruye todo germen de redención social y deja a las generaciones obreras del futuro una sociedad ignorante, esclava y triste" 59 . Por su parte, las organizaciones obreras, que habían tenido un crecimiento cuantitativo en los años 1926-1927 (el cual coincidió con la segunda apertura democrática de la década) crearon un Centro Obrero de Estudios Sociales, con el propósito de impartir conferencias y $\operatorname{cursos}^{60}$.

Lo anterior puede ser un indicio de las divergencias entre obreros y estudiantes en los años posteriores al derrocamiento de Estrada Cabrera. Mientras que la UP dirigía esfuerzos y recursos a la alfabetización, el movimiento obrero iba tras una definición política que lo acercaba al ideario comunista. Quizás por eso el prospecto de la UP incluyó la prohibición terminante, para profesores y alumnos, de "toda propaganda tendenciosa de política militante y de religión" ${ }^{61}$.

Asturias insistía en la importancia de la escuela "para los adultos que no saben leer, que ignoran que son ciudadanos" ${ }^{\text {"62 }}$. Llegó a justi-

58 Universidad Popular, Memoria de los trabajos efectuados, 18-21.

59 Miguel Ángel Asturias, "Un llamamiento a los obreros de Guatemala (en favor de la Universidad Popular)", en París 1924-1933, 19. El texto fue escrito en 1925.

60 Renate Witzel de Ciudad (coord.), "Más de 100 años del movimiento obrero", 242.

61 Universidad Popular, Prospecto de la Universidad Popular, 9.

62 Miguel Ángel Asturias "A los alumnos de la Universidad Popular de Guatemala”, en París 1924-1933, 21. Texto escrito en marzo de 1925. 
ficar el escaso fruto de la UP en sus primeros tres años de existencia argumentando que él y los otros fundadores no esperaron que las cosas cambiaran de un día para otro, y agregó: "Si es verdad que ha enseñado solamente a unos pocos, en cambio logró lo que ninguno había logrado en Guatemala: hacer trabajar por su espontánea voluntad a un ciento de personas gratuitamente en bien de la nación. Y eso es un triunfo" ${ }^{63}$.

Una crítica de la época dijo que la UP no tenía "[...] la orientación verdadera ideológica y constructiva que deben tener estos organismos, es decir, la de instituciones de enseñanza y capacitación emancipadora de las masas populares [...]"64. No obstante, reconoció la labor alfabetizadora que realizaba, la participación voluntaria de personas ajenas a la institución en el proceso y la "conquista pedagógica" alcanzada al enseñar a leer, escribir y las reglas elementales de la aritmética en un lapso de tres meses ${ }^{65}$.

Asturias regresó a Guatemala en marzo de 1928. En los primeros días de abril volvió a la UP, no como profesor, sino como conferencista. El Imparcial reconoció su "perseverancia para colaborar en actividades de educación popular” y recordó que él había sido docente del curso Lecturas Escogidas en los primeros años de la universidad ${ }^{66}$. Quizás decepcionado por lo que encontró, Asturias atribuyó a la "mentalidad atrasada del guatemalteco" la decadencia de la UP: "La Universidad Popular, llamada a los más claros destinos, poco a poco ha ido reduciendo su campo de acción, por culpa de ese mismo factor delatado" "67. El desencanto de Asturias pudo deberse a que después de cuatro años de ausencia, regresaba a un país en el cual los cambios esperados no habían tenido lugar. El panorama intelectual y político de los primeros años de

63 Miguel Ángel Asturias “¿Nueva orientación de la Universidad Popular?”, en París 1924-1933, 101102. El texto fue escrito en marzo de 1926.

64 Moisés Castro Morales y Jorge del Valle Matheu, "El movimiento reformista", 200. El texto fue escrito en 1927.

65 Moisés Castro Morales y Jorge del Valle Matheu, "El movimiento reformista", 200.

66 "Una sesión de la U. P. en honor del licdo. Asturias", El Imparcial, 2 de abril de 1928, 1; "Conferencia de Asturias en la U. P. ayer", El Imparcial, 13 de abril de 1928, 1 y 4.

67 Miguel Ángel Asturias, "Mentalidad nueva”, en París 1924-1933, 407. No obstante, Asturias guardará un recuerdo, quizás exagerado, de la labor realizada por la Universidad Popular en su primer año: "La Universidad Popular fue fundada en 1922. Contábamos con una inscripción de unas doscientas o trescientas personas, pero pronto pasaron de las dos mil [...] obreros, gente de los alrededores, hombres y mujeres. [...] Pronto hubo una sucursal de la Universidad Popular en cada provincia". Luis Harss, "Miguel Ángel Asturias, o la tierra florida", en Los nuestros (Madrid: Santillana, 2012), 84. 
la década había dado un giro de 180 grados $^{68}$. A pesar de ello, continuó defendiendo los ideales de la UP.

Los vaivenes políticos continuaron caracterizando esos años. Un nuevo cambio en el gobierno tuvo lugar a finales de 1926, propiciado por el fallecimiento de Orellana. El general Lázaro Chacón llegó a la presidencia. Aunque en sus primeros años de gobierno hubo una apertura que permitió la organización de los trabajadores urbanos, a finales de 1928 tuvo lugar una crisis que puso en duda sus concesiones. Un año después, un levantamiento militar en el Sur-Occidente del país tuvo como consecuencia el endurecimiento de las medidas contra los trabajadores y la división del movimiento obrero.

La inestabilidad política llevó a la suspensión de las garantías constitucionales, que aunada a las consecuencias de la depresión mundial y a una nueva crisis derivada de la baja en los precios del café, llevó a un resquebrajamiento del poder. A lo anterior hay que agregar la repentina enfermedad de Chacón, una advertencia que inició el movimiento de sucesión. En febrero de 1931, Jorge Ubico ganó las elecciones presidenciales. Su triunfo fue el preludio de una nueva dictadura que se prolongó 14 años.

El entusiasmo de los estudiantes universitarios para apoyar a la UP fue decayendo con el paso del tiempo. En 1932 solo siete se contaban en la lista de profesores. A pesar de ello, la UP logró enseñar a leer y a escribir a un promedio de 600 obreros al año ${ }^{69}$. En julio de ese año y como parte del cierre de espacios de disenso, un acuerdo gubernativo de Jorge Ubico derogó al que casi una década atrás había reconocido la personalidad jurídica de la Universidad Popular. Según el texto, esta había sido organizada con muchas deficiencias y no había alcanzado los fines para los que se había creado. En adelante, la Secretaría de Educación Pública se haría cargo de la reorganización de la UP ${ }^{70}$.

68 Arturo Taracena Arriola, "Miguel Ángel Asturias y la búsqueda del 'alma nacional' guatemalteca", 698.

69 Joseph A. Pitti, "Jorge Ubico and the Guatemalan Politics", 210 y 250, nota 67.

70 "Derógase un acuerdo gubernativo de fecha 30 de noviembre de 1923", 6 de julio de 1932, en Recopilación de las leyes de la República de Guatemala, 1932-1933, comp. Rosendo P. Méndez (Guatemala: Tipografía Nacional, 1935), tomo LI, 772. 
Los nuevos estatutos de la UP se aprobaron el 21 de octubre de $1932^{71}$. Los objetivos planteados siguieron ligándola a la formación de los obreros en tres ámbitos: combatir el analfabetismo, elevar el nivel moral y propagar la cultura física ${ }^{72}$. Aunque según los estatutos seguía orientada a la alfabetización y a la divulgación de conocimientos, la UP había perdido su esencia: ya no sería una institución autónoma imbuida del espíritu universitario de la década de 1920.

\section{Imagen 3}

“La Universidad se abre al pueblo”. Escultura de Rodolfo Galeotti Torres (1948). Fachada del edificio que ocupa la Universidad Popular en Guatemala

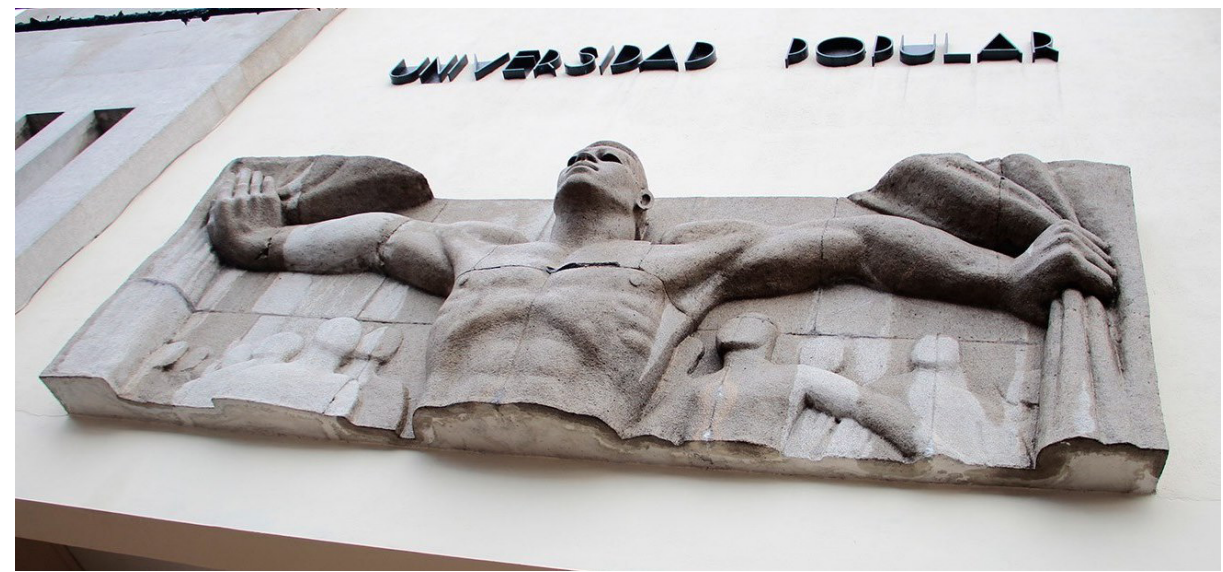

Fuente: Fotografía de Rodolfo García.

Después del triunfo de la revolución que puso fin a la dictadura de Jorge Ubico, la UP inició una nueva etapa. En 1945 la institución, que había empezado a funcionar el 12 de mayo de ese año, publicó un folleto en el que mencionó el trabajo realizado en sus centros de alfabetización y mediante su programa de divulgación cultural en su primera década de existencia. Tres de sus impulsores en los años 1920, Carlos Federico Mora, Alfonso Orantes y Marco Antonio Asturias eran parte de su Consejo Directivo ${ }^{73}$. Trece años después del cierre de la UP ordenado por Ubico, ellos perseveraban en la búsqueda de sus metas iniciales.

71 "Estatutos de la Universidad Popular de Guatemala; y acuerdo de aprobación”, 21 de octubre de 1932, en Recopilación de las leyes, tomo LI, 952-954.

72 La publicación de los estatutos constituye la única evidencia sobre la UP reformada por Ubico.

73 La Universidad Popular de Guatemala (Guatemala: Unión Tipográfica, 1945), 3. 


\section{A MODO DE CIERRE}

El bregar de la UP en su primera década debe entenderse a la luz de la confluencia de tres procesos. Como ha quedado dicho, el primero de ellos fue la participación estudiantil en el derrocamiento de Estrada Cabrera, la cual reveló al grupo sus posibilidades de acción. El segundo está constituido por las propuestas de reforma que impulsaron la Reforma Universitaria de Córdoba y Vasconcelos, asumidas y re-elaboradas por el propio grupo; y el tercer proceso, que resultó determinante, es la combinación de los graves atrasos estructurales del país con las prácticas políticas profundamente conservadoras de la élite dirigente. En otras palabras, es un momento en el que se vinculan condiciones internacionales favorables con una élite secundaria receptiva, pero que encuentra su freno en las condiciones económicas, políticas y sociales imperantes.

En ese entorno, el grupo de estudiantes universitarios, la llamada Generación de 1920, impregnado de un activismo romántico, se propuso impulsar un modelo alternativo de educación que rebasara los planteamientos y la escasa cobertura de la educación de adultos servida por el Estado. De ahí que concibiera un programa que incluía conocimientos básicos de lecto-escritura y aritmética, complementados con cultura ciudadana.

La experiencia se extendió a varios lugares del interior del país, con resultados disímiles. Una de sus grandes falencias fue ignorar que la población rural era mayoritariamente indígena y monolingüe, atrapada en un sistema productivo que no necesitaba trabajadores alfabetas. Por otro lado, la población meta urbana de la UP, constituida básicamente por obreros y artesanos, siguió su propia agenda y buscó opciones políticas que la alejaron de la propuesta inicial de la universidad. Los objetivos e intereses de ambos grupos tomaron diferentes caminos.

En suma, el espíritu continental de la Reforma de Córdoba y de Vasconcelos se identifica claramente en las acciones emprendidas por esta generación. Su interpretación de la extensión universitaria, subtema que concitó gran interés en Guatemala, se plasmó en este esfuerzo 
de alfabetización de adultos, cuyos alcances y limitaciones estuvieron dados por el entorno sociopolítico de entonces.

\section{BibLIOGRAFÍA}

\section{Fuentes primarias}

\section{Publicaciones periódicas}

El Imparcial (Guatemala): 1 marzo 1923; 2 marzo 1923; 6 marzo 1923; 8 marzo 1923; 13 marzo 1923; 2 abril 1928; 13 abril 1928; 1 marzo 1963.

Studium. Revista mensual, órgano de la Asociación de Estudiantes Universitarios de Guatemala: Vol. 1, No. 7 (noviembre-diciembre 1921); Vol. 2, Nos. 2 y 3 (febrero-marzo 1923).

\section{Documentos oficiales}

Guatemala. Secretaría de Instrucción Pública. Memoria de la Secretaría de Instrucción Pública presentada a la Asamblea Nacional Legislativa en 1907. Guatemala: Tipografía Nacional, 1907.

Guatemala. Secretaría de Instrucción Pública. Programas detallados para las escuelas de enseñanza complementaria de la República de Guatemala. Guatemala: Tipografía Nacional, 1922.

Guatemala. Ministerio de Fomento. Censo de la población de la República levantado el 28 de agosto de 1921, parte I. Guatemala: Talleres Gutenberg, 1924.

Guatemala. Secretaría de Educación Pública. Memoria de la Secretaría de Educación Pública, presentada a la Asamblea Nacional Legislativa en sus sesiones ordinarias de 1924. Guatemala: Tipografía Nacional, 1924.

\section{Documentos relativos a la Universidad Popular}

Universidad Popular. Prospecto de la Universidad Popular. Guatemala: Tipografía Nacional, 1923. . Guía de profesores adherentes. Guatemala: Tipografía Nacional, 1923. 
. Guía de profesores de curso. Guatemala: Tipografía Nacional, 1923.

- Reglamento y programas para las escuelas de analfabetos de la Universidad Popular de Guatemala. Guatemala: s.e., 1923.

- Memoria de los trabajos efectuados en la Universidad Popular de Guatemala, hasta el 31 de diciembre de 1924. Guatemala: Talleres Selca, 1924.

"Derógase un acuerdo gubernativo de fecha 30 de noviembre de 1923", 6 de julio de 1932. En Recopilación de las leyes de la República de Guatemala, 1932-1933, compilado por Rosendo P. Méndez. Guatemala: Tipografía Nacional, 1935, tomo LI, 772.

"Estatutos de la Universidad Popular de Guatemala; y acuerdo de aprobación", 21 de octubre de 1932. En Recopilación de las leyes de la República de Guatemala, 1932-1933, compilado por Rosendo P. Méndez. Guatemala: Tipografía Nacional, 1935, tomo LI, 952954.

La Universidad Popular de Guatemala. Guatemala: Unión Tipográfica, 1945.

\section{Testimonios}

Asturias, Miguel Ángel. París 1924-1933. Periodismo y creación literaria, coordinado por Amos Segala. Madrid: ALLCA XX, 1997.

Quintana, Epaminondas. Historia de la generación de 1920. Guatemala: Tipografía Nacional, 1971.

\section{Otros documentos}

"Resoluciones del Primer Congreso Internacional de Estudiantes reunido en la ciudad de México del 20 de setiembre al 8 de octubre de 1921". Repertorio Americano Vol. 3 No. 27 (27 de febrero de 1922): 375-376.

"Universidad Popular: tres consideraciones sobre la universidad clásica cerrada". Cultura Vol. 1 No. 3 (septiembre 1922): 1-4.

\section{Fuentes secundarias}

Álvarez Aragón, Virgilio. Conventos, aulas y trincheras: universidad y movimiento estudiantil en Guatemala, tomo I. Guatemala: FLACSO, 2002. 
Burkholder, Mark A., Monica Rankin y Lyman L. Johnson. Exploitation, Inequality and Resistance: A History of Latin America since Columbus. New York y Oxford: Oxford University Press, 2018.

Castro Morales, Moisés y Jorge del Valle Matheu. "El movimiento reformista en las universidades nacionales y populares de Centro América". En La reforma universitaria, tomo II, compilado por Gabriel del Mazo. La Plata: Centro de Estudiantes de Ingeniería, 1941, 198-201.

Carrillo Padilla, Lorena. Luchas de las guatemaltecas del siglo XX. Mirada al trabajo y la participación política de las mujeres. Guatemala: Ediciones del Pensativo, 2004.

Cazali Ávila, Augusto. Historia de la Universidad de San Carlos de Guatemala: época republicana, 1821-1944, $2^{\mathrm{a}}$ ed. Guatemala: Editorial Universitaria, 2001.

Guibert, Rita. "Miguel Angel Asturias". En Seven Voices: Seven Latin American Writers Talk to Rita Guibert. New York: Alfred A. Knopf, 1974, 119-180.

Harss, Luis. "Miguel Ángel Asturias, o la tierra florida". En Los nuestros (Madrid: Santillana, 2012), 77-111.

Jones, J. Bascom, ed. El libro azul de Guatemala, 1915. New Orleans: Searcy \& Pfaff, 1915.

Melgar Bao, Ricardo. "Las universidades populares en América Latina 1910-1925”. Estudios No. 11-12 (1999): 41-57.

Phillips, Eugenia A. "The Development of Modern Education in Guatemala”. Tesis de Maestría, University of Southern California, 1927.

Pitti, Joseph A. "Jorge Ubico and the Guatemalan Politics in the 1920's". Tesis de Doctorado, University of New Mexico, 1975.

Rendon, Mary Catherine. "Manuel Estrada Cabrera, Guatemalan President, 1898-1920”. Tesis de Doctorado, Oxford University, 1988.

Taracena Arriola, Arturo. "Miguel Ángel Asturias y la búsqueda del 'alma nacional' guatemalteca. Itinerario político, 1920-1923”. En Miguel Ángel Asturias. París 1924-1933. Periodismo y creación literaria, coordinado por Amos Segala. Madrid: ALLCA XX, 1997, 679-708.

."El movimiento obrero y los estudiantes universitarios, 19201927’. Perspectiva No. 8 (enero-junio 1986): 65-77. 
Tünnermann Bernheim, Carlos. "La reforma universitaria de Córdoba”. Educación Superior y Sociedad Vol. 9 No. 1 (1998): 103-127. Witzel de Ciudad, Renate (coord.). Más de 100 años del movimiento obrero urbano en Guatemala, tomo I. Guatemala: Asíes, 1991.

Para citar este artículo: González Sandoval, Leticia. "Los estudiantes universitarios guatemaltecos y su proyección social: la Universidad Popular (1923-1932)", Historia Caribe Vol. XVI No. 38 (Enero-Junio 2021): 137-165. DOI: https://doi.org/10.15648/hc.38.2021.2814 